\title{
Approaches for Preventing Healthcare-Associated Infections: Go Long or Go Wide?
}

\author{
Edward Septimus, MD; ${ }^{1}$ Robert A. Weinstein, MD; ${ }^{2}$ Trish M. Perl, MD, MSc; ${ }^{3}$ \\ Donald A. Goldmann, MD; ${ }^{4,5}$ Deborah S. Yokoe, MD, $\mathrm{MPH}^{6}$
}

In this issue, the continuing "A Compendium of Strategies to Prevent Healthcare-Associated Infections in Acute Care Hospitals: 2014 Updates" series presents updated recommendations for preventing central line-associated bloodstream infections ${ }^{1}$ and preventing transmission and infection due to methicillin-resistant Staphylococcus aureus. ${ }^{2}$ During revision of these articles, several reviewers raised a critical question: What is the relative effectiveness (and cost-effectiveness) of vertical versus horizontal approaches to infection prevention? As multidrug-resistant organisms such as extendedspectrum $\beta$-lactamase-producing and carbapenem-resistant Enterobacteriaceae emerge and spread, it will become increasingly important to understand the relative benefits and costs of pathogen-specific screening and intervention strategies compared with reliable application of more "generic" methods to mitigate transmission and infection.

Over the last decade, the general approaches to healthcareassociated infection (HAI) prevention have taken two conceptually different paths: (1) vertical approaches that aim to reduce colonization, infection, and transmission of specific pathogens, largely through use of active surveillance testing (AST) to identify carriers, followed by implementation of measures aimed at preventing transmission from carriers to other patients, and (2) horizontal approaches that aim to reduce the risk of infections due to a broad array of pathogens through implementation of standardized practices that do not depend on patient-specific conditions. Examples of horizontal infection prevention strategies include minimizing the unnecessary use of invasive medical devices, enhancing hand hygiene, improving environmental cleaning, and promoting antimicrobial stewardship (Table 1). ${ }^{3}$ Although vertical and horizontal approaches are not mutually exclusive and are often intermixed, some experts believe that the horizontal approach under usual endemic situations may offer the best overall value given the diversity of microorganisms that can cause HAIs and the constrained resources available for infection prevention efforts. When informed by local knowledge of microbial epidemiology and ecology and supported by a strong quality improvement program, this strategy allows healthcare facilities to focus on approaches that target all rather than selected organisms in the absence of an organismspecific epidemic.

In addition to comparing the strength of evidence supporting each approach, it is also important to take into account financial costs and potential consequences associated with various infection prevention strategies, including the impact on hospital personnel effort and on aspects of patient care; for example, placing patients on isolation precautions may lead to fewer healthcare provider visits. ${ }^{4-6}$ These comparisons are difficult to make because of conflicting study results, at least partly reflecting the heterogeneity of study designs and settings (ie, where the prevalence of the target pathogen ranges from rare to endemic to epidemic) and the paucity of high-quality cost-effectiveness analyses that are needed to estimate the economic impact of specific HAI prevention interventions.

\section{VERTICAL APPROACHES TO PREVENT HAIS}

Vertical approaches utilize activities that are directed at a single pathogen or specific groups of pathogens and are often based on the results of AST. The rationale for AST is that multidrug-resistant organisms (MDROs) such as methicillinresistant Staphylococcus aureus (MRSA), vancomycin-resistant enterococci (VRE), multidrug-resistant (MDR) gram-negative organisms, and Clostridium difficile share several epidemiological features: colonization can precede infection, transmission can occur by direct patient contact or indirect contact with contaminated equipment or environmental surfaces, the number of asymptomatic "source" patients greatly exceeds

Affiliations: 1. Texas A\&M Health Science Center College of Medicine, Houston, Texas, and Hospital Corporation of America, Nashville, Tennessee; 2. Stroger Hospital and Rush University Medical Center, Chicago, Illinois; 3. Johns Hopkins University School of Medicine, Baltimore, Maryland; 4. Institute for Healthcare Improvement, Cambridge, Massachusetts; 5. Boston Children's Hospital and Harvard Medical School, Boston, Massachusetts; 6. Brigham and Women's Hospital and Harvard Medical School, Boston, Massachusetts.

Received March 17, 2014; accepted March 18, 2014; electronically published June 9, 2014.

Infect Control Hosp Epidemiol 2014;35(7):797-801

(C) 2014 by The Society for Healthcare Epidemiology of America. All rights reserved. 0899-823X/2014/3507-0003\$15.00. DOI: 10.1086/676535 
TABLE 1. Preventing Healthcare-Associated Infections: Examples of Vertical and Horizontal Approaches

Vertical approaches reduce risk of infections due to specific pathogens:

- Active surveillance testing to identify asymptomatic carriers

- Contact precautions for patients colonized or infected with specific organisms

- Decolonization of patients colonized or infected with specific organisms

Horizontal approaches reduce risk of a broad range of infections and are not pathogen specific:

- Standard precautions (eg, hand hygiene)

- Universal use of gloves or gloves and gowns

- Universal decolonization (eg, chlorhexidine gluconate bathing)

- Antimicrobial stewardship

- Environmental cleaning and disinfection

sourCE. Modified from Wenzel and Edmond.

the number of infected patients, and asymptomatic carriers can serve as the reservoir for spread to other patients. AST is used to identify patients who are carriers of these target pathogens so that these patients can be isolated from noncarriers and, in some situations, can undergo decolonization in order to eradicate pathogen carriage.

This approach has been most intensely studied for prevention of MRSA transmission and infection. More than 100 observational studies have evaluated the use of MRSA AST to target MRSA carriers for contact precautions, with or without supplemental decolonization. The effectiveness of AST in preventing MRSA transmission and infection continues to be controversial, and studies on this topic have yielded varying conclusions. The Dutch national strategy for MRSA prevention and control is based on a very proactive and aggressive approach called "search and destroy," aimed at identifying all hospitalized MRSA carriers. "This process requires AST of persons with epidemiologic links to MRSA carriers (eg, other patients hospitalized in geographic proximity, healthcare providers, family members) as well as isolation of MRSA carriers and cohorting of staff who care for MRSA carriers. However, critics point out that aggressive strategies such as these may only be cost effective in parts of the world with very low MRSA prevalence. Jain et $\mathrm{al}^{8}$ described a nationwide intervention in Veterans Affairs acute care hospitals that included MRSA AST and contact precautions for MRSA carriers, improved compliance with hand hygiene, and an institutional culture change that was temporally associated with a large decline in infections caused by MRSA as well as other pathogens. In contrast, in a concurrently published study, Huskins et $\mathrm{al}^{9}$ described a multicenter cluster-randomized, controlled trial in intensive care units (ICUs) that demonstrated that an intervention involving MRSA AST plus universal gloving until a patient's colonization status was known to be negative did not impact rates of MRSA colonization or infection. A recent review of MRSA screening strategies concluded that the overall quality of evidence to support the use of AST to prevent healthcare-associated MRSA infections was low. ${ }^{10}$

The data assessing the utility of AST for preventing MDR gram-negative bacilli transmission and infection are even more controversial, and supporting evidence is largely based on studies in which AST and a number of additional control measures are simultaneously implemented to control carbapenem-resistant Enterobacteriaceae (CRE) transmission in hospital and regional outbreak settings. ${ }^{11-16}$ AST is recommended by the Centers for Disease Control and Prevention as a strategy to control CRE transmission. ${ }^{17}$

\section{HORIZONTAL APPROACHES TO}

\section{PREVENT HAIS}

Hand hygiene is acknowledged as one of the most important horizontal strategies for preventing HAIs. Despite this, published rates of hand hygiene adherence average about $40 \% .^{18}$ There are dramatic examples of the impact of hand hygiene improvements on the risk of infections associated with resistant organisms. Jarlier and colleagues demonstrated that a $35 \%$ decrease in MRSA infections in French ICUs correlated with an increase in hand hygiene. ${ }^{19}$ In addition, some studies have suggested that universal gloving can also reduce transmission of MDROs by preventing contamination of the hands of healthcare personnel, ${ }^{20}$ and universal gloving has been associated with significant reductions in all-cause bacteremia and central line-associated bloodstream infections in acute pediatric units during respiratory syncytial virus season. ${ }^{21}$ The impact of universal gowning and gloving remains controversial. A recently published cluster-randomized trial involving 20 adult ICUs assigned to either routine use of contact precautions for patients with known pathogen carriage or use of gowns and gloves for all patient contacts concluded that universal gowning and gloving did not result in a significant difference in the primary study outcome of MRSA or VRE acquisition but was associated with a lower risk of MRSA acquisition alone. ${ }^{22}$ The investigators found that use of gloves and gowns was associated with fewer healthcare personnel visits but improved hand hygiene compliance in both control and intervention ICUs, and no difference in the risk of adverse events was noted between control and intervention patients. Because of the associated expense and healthcare personnel time required for universal usage of gloves and gowns, this strategy is unlikely to be feasible outside of the ICU setting. 
Decolonization of all patients in high-risk settings using topical chlorhexidine gluconate (CHG) is another horizontal strategy that has received increased attention. CHG bathing has been shown to decrease the bioburden of microorganisms on the patient, the environment, and the hands of healthcare personnel. ${ }^{23}$ During 2013, four randomized cluster trials were published evaluating the effectiveness of CHG bathing in preventing HAIs among ICU patients. Climo et $\mathrm{a}^{24}$ performed a cluster-crossover study and found that daily CHG bathing of adult ICU patients significantly reduced their risks for developing hospital-acquired bloodstream infections, including central line-associated bloodstream infection, and acquiring VRE. Using a similar study design, Milstone et $\mathrm{a}^{25}$ reported that $\mathrm{CHG}$ bathing was associated with a significant reduction in bloodstream infections among pediatric ICU patients compared to standard bathing. Two additional studies included a comparison of horizontal and vertical approaches. Huang et $\mathrm{al}^{26}$ compared three approaches to MRSA prevention among patients in 74 adult ICUs (the REDUCE MRSA study). Vertical approaches consisting of AST with and without targeted decolonization of MRSA carriers with CHG bathing and intranasal mupirocin were compared to a more horizontal approach involving universal decolonization of all ICU patients regardless of MRSA status. The investigators found that universal decolonization of all ICU patients was associated with the largest reduction in all-cause bloodstream infection (44\%) and MRSA clinical culture rates (37\%). Finally, Derde et $\mathrm{al}^{27}$ demonstrated that improved hand hygiene plus universal CHG bathing reduced acquisition of MDROs including MRSA and showed that in a setting where high levels of adherence to hand hygiene and CHG bathing were sustained, the addition of AST (either rapid or conventional testing) and isolation of carriers did not further reduce MDRO acquisition rates. These four recent studies add to growing evidence that in endemic settings, vertical strategies that involve AST and isolation may not be as effective as more horizontal approaches utilizing hand hygiene and universal decolonization using CHG bathing with or without intranasal mupirocin. It should be noted that although these studies evaluate interventions applied to all patients in highrisk settings (ie, ICUs), the use of intranasal mupirocin specifically targets Staphylococcus aureus, one out of many potential pathogens.

Decolonization using CHG bathing to prevent CRE transmission and infection has been utilized along with other infection control strategies in outbreak settings. ${ }^{14,15,28}$

Selective digestive tract decontamination (SDD) is a horizontal strategy aimed at eradicating carriage of pathogens to prevent subsequent respiratory tract infections. SDD typically consists of topical application of antimicrobial agents (eg, polymyxin, tobramycin, and amphotericin) in the oropharynx and into the gastrointestinal tract through a nasogastric tube. Some SDD regimens also include an initial course of parenteral antimicrobials (eg, cefotaxime) in addition to topical agents. A systematic review included data from 36 ran- domized controlled trials and concluded that SDD regimens consisting of combinations of topical and systemic antimicrobial agents reduce the risk for respiratory tract infection and overall mortality among adults receiving ICU care. ${ }^{29} \mathrm{De}-$ spite supportive evidence, SDD has not been widely adopted as an infection prevention strategy in the United States mainly because of concerns regarding the potential risk for increasing antimicrobial resistance. Additional studies are needed to assess the impact of SDD on the epidemiology of antimicrobial resistance among gram-negative bacteria, particularly in a setting where the prevalence of MDR gram-negative bacilli is relatively high.

Misuse and overuse of antimicrobials facilitate the development of MDROs, making antimicrobial stewardship (AS) an important horizontal HAI prevention strategy that can complement other approaches. AS efforts ideally involve interdisciplinary collaboration aimed at providing prudent and appropriate antimicrobial use for patients across the continuum of care. AS has been shown to be critical in reducing rates of HAIs and the risks of other adverse events resulting from exposure to antimicrobial agents. Improving antimicrobial prescribing practices in conjunction with other infection prevention strategies has been effective in reducing Clostridium difficile infection (CDI) risk in outbreak and nonoutbreak settings. ${ }^{30,31}$ Additional research is needed to better quantify the impact of AS on MDRO risks.

Adequate cleaning of the healthcare environment is an important horizontal HAI prevention strategy. Evidence is increasing that contaminated surfaces play an important role in the transmission of several key pathogens including Clostridium difficile, VRE, MRSA, Acinetobacter baumannii, and norovirus. All of these organisms can persist from hours to days in the environment. Healthcare personnel can contaminate their hands not only through direct contact with a colonized or infected patient but also by touching contaminated hospital surfaces. Furthermore, recent studies have linked the risk of transmission to the colonization status of prior room occupants for MRSA and VRE, ${ }^{32,33} \mathrm{CDI},{ }^{34}$ and multidrugresistant Acinetobacter baumannii and Pseudomonas aeruginosa. ${ }^{35}$ Most important, improved environmental cleaning has led to reduced risk of VRE and $C$. difficile transmission. ${ }^{36}$

\section{CONCLUSION}

Reliable implementation is critical for either vertical or horizontal strategies. As new data emerge, prevention measures known to be effective should be integrated into care, applied reliably, and sustained. ${ }^{37}$ Quality improvement programs can play an important role in facilitating change and ensuring that implementation and intra-institutional spread respect local contexts. Unintended consequences should be anticipated and monitored.

It is important to recognize that there is no "one-size-fitsall" approach to improving practices. Local contextual factors matter, and specific components of the implementation plan 
should be tested (Plan-Do-Study-Act [PDSA] cycles) and amended using standard quality improvement methods, such as those specified in the Model for Improvement. ${ }^{38}$

Our current ability to adequately compare the cost effectiveness of horizontal and vertical HAI prevention strategies or combinations of these strategies across healthcare settings is severely limited by the absence of robust data. Given the evolving epidemiology of MDROs and the complexity of managing the multiplicity of epidemiologically important pathogens across heterogeneous healthcare settings, however, we recommend (1) using robust quality improvement methods to ensure reliable performance of basic infection prevention practices known to mitigate transmission of MDROs and the infections they cause; (2) ensuring adherence to evidencebased universally applied HAI prevention strategies including hand hygiene, antimicrobial stewardship, and adequate environmental cleaning; (3) applying other evidence-based, horizontal strategies such as universal decolonization in settings where benefits are likely to outweigh risks and costs; and (4) using AST and other vertical approaches selectively when epidemiologically important pathogens are newly emerging and rare to a given institution or region or to control outbreaks of specific pathogens.

\section{ACKNOWLEDGMENTS}

Financial support. T.M.P. reports receiving research funding from Merck.

Potential conflicts of interest. All other authors report no conflicts of interest relevant to this article.

Address correspondence to Edward Septimus, MD, 4257 Albans, Texas A\&M Health Science Center, Houston, TX 77005 (eseptimus@gmail.com).

\section{REFERENCES}

1. Marschall J, Mermel LA, Fakih M, et al. Strategies to prevent central line-associated bloodstream infections in acute care hospitals: 2014 update. Infect Control Hosp Epidemiol 2014;35(7): 753-771 (in this issue).

2. Calfee DP, Salgado CD, Milstone AM, et al. Strategies to prevent methicillin-resistant Staphylococcus aureus transmission and infection in acute care hospitals: 2014 update. Infect Control Hosp Epidemiol 2014;35(7):772-796 (in this issue).

3. Wenzel RP, Edmond MB. Infection control: the case for horizontal rather than vertical interventional programs. Int J Infect Dis 2010;14(suppl 4):\$3-S5.

4. Morgan DJ, Pineless LL, Shardell M, et al. The effect of contact precautions on healthcare worker activity in acute care hospitals. Infect Control Hosp Epidemiol 2013;34(1):69-73.

5. Morgan DJ, Diekema DJ, Sepkowitz K, Perencevich EN. Adverse outcomes associated with contact precautions: a review of the literature. Am J Infect Control 2009;37(2):85-93.

6. Stelfox HT, Bates DW, Redelmeier DA. Safety of patients isolated for infection control. JAMA 2003;290(14):1899-1905.

7. Vos MC, Behrendt MD, Melles DC, et al. 5 years of experience implementing a methicillin-resistant Staphylococcus aureus search and destroy policy at the largest university medical center in the Netherlands. Infect Control Hosp Epidemiol 2009;30(10): 977-984.

8. Jain $\mathrm{R}$, Kralovic SM, Evans ME, et al. Veterans affairs initiative to prevent methicillin-resistant Staphylococcus aureus infections. N Engl J Med 2011;364:1419-1430.

9. Huskins WC, Huckabee CM, O'Grady NP, et al. Intervention to reduce transmission of resistant bacteria in intensive care. $N$ Engl J Med 2011;364:1407-1418.

10. Glick SB, Samson DJ, Huang E, et al. Screening for methicillinresistant Staphylococcus aureus (MRSA). Comparative Effectiveness Review no. 102. AHRQ Publication no. 13-EHC043-EF. Rockville, MD: Agency for Healthcare Research and Quality; June 2013. http://www.effectivehealthcare.ahrq.gov/reports /final.cfm.

11. Ben-David D, Maor Y, Keller N, et al. Potential role of active surveillance in the control of a hospital-wide outbreak of carbapenem-resistant Klebsiella pneumoniae infection. Infect Control Hosp Epidemiol 2010;31:620-626.

12. Calfee D, Jenkins SG. Use of active surveillance cultures to detect asymptomatic colonization with carbapenem-resistant Klebsiella pneumoniae in intensive care unit patients. Infect Control Hosp Epidemiol 2008;29:966-968.

13. Kochar S, Sheard T, Sharma R, et al. Success of an infection control program to reduce the spread of carbapenemase-producing Klebsiella pneumoniae. Infect Control Hosp Epidemiol 2009;30:447-452.

14. Munoz-Price LS, Hayden MK, Lolans K, et al. Successful control of an outbreak of Klebsiella pneumoniae carbapenemase-producing $K$. pneumoniae at a long-term acute care hospital. Infect Control Hosp Epidemiol 2010;31:341-347.

15. Palmore TN, Henderson DK. Managing transmission of carbapenem-resistant Enterobacteriaceae in healthcare settings: a view from the trenches. Clin Infect Dis 2013;57:1593-1599.

16. Schwaber MJ, Carmeli Y. An ongoing national intervention to contain the spread of carbapenem-resistant Enterobacteriaceae. Clin Infect Dis 2014;58:697-703.

17. Centers for Disease Control and Prevention. CRE toolkit: guidance for control of carbapenem-resistant Enterobacteriaceae (CRE). 2012. http://www.cdc.gov/hai/organisms/cre/cre-toolkit /index.html. Accessed March 13, 2014.

18. Erasmus V, Daha TJ, Brug H, et al. Systematic review of studies on compliance with hand hygiene. Infect Control Hosp Epidemiol 2010;31:283-294.

19. Jarlier V, Trystram D, Brun-Buisson $C$, et al. Curbing methicillin-resistant Staphylococcus aureus in 38 French hospitals through a 15-year institutional control program. Arch Intern Med 2010;170:552-559.

20. Tenorio AR, Badri SM, Sahgal NB, et al. Effectiveness of gloves in the prevention of hand carriage of vancomycin resistant enterococcus species by health care workers after patient care. Clin Infect Dis 2001;32:826-829.

21. Yin J, Schweizer ML, Herwaldt LA, et al. Benefits of universal gloving on hospital-acquired infections in acute care pediatric units. Pediatrics 2013;131:e1515-e1520.

22. Harris AD, Pineless $L$, Belton $B$, et al. Universal glove and gown use and acquisition of antibiotic-resistant bacteria in the ICU: a randomized trial. JAMA 2013;310(15):1571-1580.

23. Vernon MO, Hayden MK, Trick WE, et al. Chlorhexidine gluconate to cleanse patients in a medical intensive care unit: the effectiveness of source control to reduce the bioburden of van- 
comycin-resistant enterococci. Arch Intern Med 2006;166:306312.

24. Climo MW, Yokoe DS, Warren DK, et al. Effect of daily chlorhexidine bathing on hospital-acquired infection. $N$ Engl J Med 2013;368:533-542.

25. Milstone AM, Elward A, Song X, et al. Daily chlorhexidine bathing to reduce bacteraemia in critically ill children: a multicentre, cluster-randomised, crossover trial. Lancet 2013;381:1099-1106.

26. Huang SS, Septimus E, Kleinman K, et al. Targeted versus universal decolonization to prevent ICU infection. $N$ Engl J Med 2013;368:2255-2265.

27. Derde LPG, Cooper BS, Goossens $\mathrm{H}$, et al, on behalf of the MOSAR WP3 Study Team. Interventions to reduce colonisation and transmission of antimicrobial-resistant bacteria in intensive care units: an interrupted time series study and cluster randomised trial. Lancet Infect Dis 2014;(14):31-39.

28. Munoz-Price LS, De La Cuesta C, Adams S, et al. Successful eradication of a monoclonal strain of Klebsiella pneumoniae during a $K$. pneumoniae carbapenemase-producing $K$. pneumoniae outbreak in a surgical intensive care unit in Miami, Florida. Infect Control Hosp Epidemiol 2010;31:1074-1077.

29. D'Amico R, Pifferi S, Torri V, Brazzi L, Parmelli E, Liberati A. Antibiotic prophylaxis to reduce respiratory tract infections and mortality in adults receiving intensive care. Cochrane Database Syst Rev 2009;(4):CD000022. doi:10.1002/14651858.CD000022 .pub3.

30. Valiquette L, Cossette B, Garant MP, Diab H, Pepin J. Impact of a reduction in the use of high-risk antibiotics on the course of an epidemic of Clostridium difficile-associated disease caused by the hypervirulent NAP1/027 strain. Clin Infect Dis 2007; 45(suppl 2):S112-S121.
31. Carling P, Fung T, Killion A, Terrin N, Barza M. Favorable impact of a multidisciplinary antibiotic management program conducted during 7 years. Infect Control Hosp Epidemiol 2003; 24(9):699-706.

32. Huang SS, Datta R, Platt R. Risk of acquiring antibiotic-resistant bacteria from prior room occupants. Arch Intern Med 2006;166: 1945.

33. Datta R, Platt R, Yokoe DS, et al. Environmental cleaning interventions and risk of acquiring multidrug resistant organisms from prior room occupants. Arch Intern Med 2011;171(6):491494.

34. Shaughnessy MK, Micielli RL, DePestel D, et al. Evaluation of hospital room assignment and acquisition of Clostridium difficile infection. Infect Control Hosp Epidemiol 2011;32:201-206.

35. Nseir S, Blazejewski C, Lubret R, et al. Risk of acquiring multidrug-resistant Gram-negative bacilli from prior room occupants in the intensive care unit. Clin Micro Infect 2011;17:12011208.

36. Hayden MK, Bonten MJ, Blom DW, Lyle EA, van de Vijver DA, Weinstein RA. Reduction in acquisition of vancomycin-resistant enterococcus after enforcement of routine environmental cleaning measures. Clin Infect Dis 2006;42(11):1552-1560.

37. Sandora TJ, Goldmann DA. Preventing lethal hospital outbreaks of antibiotic-resistant bacteria. N Engl J Med 2012;367:21682170 .

38. Langley GL, Nolan KM, Nolan TW, Norman CL, Provost LP. The Improvement Guide: A Practical Approach to Enhancing Organizational Performance. 2nd ed. San Francisco: Jossey-Bass, 2009. 